\title{
Differences of Processing Difficulty Between Phrases and Words in School-Aged Children Who Stutter
}

\author{
Saburo TAKAhashi ${ }^{1, *}$ and Tomohiko Ito ${ }^{2}$ \\ ${ }^{1}$ United Graduate School of Education, Tokyo Gakugei University, Japan \\ ${ }^{2}$ Tokyo Gakugei University, Japan
}

\begin{abstract}
Takahashi and Ito (2012) suggested that difficulty in clause-level processing does not affect the frequency of stuttering in school-aged children who stutter. This suggests that they do not experience difficulty with processing of syntactic structures. If this is the case, they may not experience difficulty with processing of phrases, which are smaller syntactic units than clauses, and phrase-level processing will not be more difficult than word-level processing. It is predicted that the frequency of stuttering of noun phrases will not be significantly higher than that of compound nouns. Results show that the frequency of stuttering of noun phrases was not significantly higher than that of compound nouns. There were no significant difference in the loci of stuttering between noun phrases and compound nouns. The results suggest that phrase-level processing is not more difficult than wordlevel processing, and that school-aged children who stutter do not experience difficulty with processing syntactic structures.
\end{abstract}

Key Words: stuttering, children who stutter, linguistic unit, noun phrase, compound noun

\section{Introduction}

Although the cause of stuttering is still imperfectly understood, there have been a number of studies investigating linguistic processing in children and adults who stutter. It has been observed that the frequency and the loci of stuttering is closely related to syntactic structures in young children who stutter. For example, stuttering frequency is correlated with increases in syntactic complexity (Buhr \& Zebrowski, 2009; Gaines, Runyan, \& Meyers, 1991; Ratner \& Sih, 1987). Moreover, stuttering frequently occurs on the first word of syntactic structures such as clauses or phrases (Bernstein, 1981; Bloodstein \& Grossman, 1981). Based on these findings, it appears that young children who stutter experience difficulty with processing of syntactic structures. If this is the case, do school-aged children also experience difficulty with processing of syntactic structures such as clauses or

\footnotetext{
* Corresponding Author

Mailing Address: 4-1-1 Nukuikitamachi, Koganei-shi, Tokyo 184-8501, Japan

E-mail Address: sablow@cg8.so-net.ne.jp

Received October 15, 2012, Accepted April 19, 2014

DOI: $10.6033 /$ specialeducation.3.11
}

phrases?

As for school-aged children who stutter, Takahashi and Ito (2012) investigated whether difficulty in processing of syntactic structure is closely related to the frequency of stuttering using sentences with relative and coordinate clauses. The structure of a sentence with a relative clause is different from that with a coordinate clause, and the former has a more complex structure than the latter. Therefore, it is expected that the frequency of stuttering in sentences with a relative clause will be significantly higher than in sentences with a coordinate clause. However, the frequency of stuttering in sentences with a relative clause was not significantly higher than in sentences with a coordinate clause. These results suggest that difficulty in clause-level processing does not significantly affect the frequency of stuttering in school-aged children who stutter. Thus, they may not have trouble with processing of syntactic structures. If this is the case, they also may not experience difficulty with processing of phrases, which are smaller syntactic units than clauses. Therefore, processing of phrases will not be any more difficult than processing of words in school-aged children who stutter. It is predicted that the frequency of stuttering in noun phrases would not be significantly higher than in 
compound nouns. However, few studies have compared the difficulties of noun phrases and compound nouns. The purpose of this study was to investigate whether the frequency of stuttering in noun phrases was significantly higher than in compound nouns for Japanese school-aged children who stutter.

\section{Methods}

\section{Participants}

The participants were 14 elementary school-aged children who stutter (13 boys and 1 girl). They were all native speakers of Japanese, and all were attending speech and hearing classes in elementary schools in Tokyo. Their ages ranged from 6 to 11, with a mean age of 9.3. Permissions for the children's participation were obtained from their parents.

\section{Stimulus Phrases and Words}

Table 1 shows stimulus phrases and words. Fourteen noun phrases and compound nouns were used. In order to control the initial segments, a number of moras and the head in noun phrases and compound nouns, phrases such as "ushi no takusii" and compound nouns such as "usagi takusii," were used. The stimulus phrases and words were divided into two groups. Group A contained 7 phrases and words whose head was "taxi," and group B included 7 phrases and words whose head was "bus." In the practice task, stimulus phrases and words which were not used in the experimental task were used.

\section{Procedures}

The children were tested individually in a room at their schools. We conducted a noun phrase task and a compound noun task in the present study. Half of the participants began with a noun phrase task followed by a compound noun task, and the other half began with a compound noun task followed by a noun phrase task. A practice task was used before the experimental task. Stimulus pictures which elicited stimulus phrases and words were presented on the screen of a notebook computer.

The procedure of the noun phrase task was as follows: First, each of the stimulus pictures of Group A was presented, and participants were taught all 7 stimulus phrases one by one. Participants were required to produce the stimulus phrases as fast as possible when the stimulus pictures were presented. Second, we presented each of the stimulus pictures of Group B, and all 7 stimulus phrases were taught to the participants. They were required to produce the stimulus phrase as well. The compound noun task was run in a similar manner. Responses were recorded using a headset (Logicool H530) and notebook computer, and HDD video recorder (Victor SRDVM700) through the camera (Victor TK-S686).

\section{Method of Analysis}

Stuttering events were defined as repetitions of a sound or a syllable, and prolongations and blocks, in accordance with Wingate's behavioral definition of stuttering (Wingate, 1964). The frequency of stuttering was calculated by dividing the total number of elements stuttered by the total number of elements, and multiplying the results by 100 .

Reliability was scored by the Sander Agreement Index (Sander, 1961), i.e., the rate of agreement was calculated by dividing the number of agreements by the total number of agreements plus disagreements, and multiplying the number derived by 100 . Audiotapes from about $25 \%$ of the participants, selected

Table 1 Stimulus Phrases and Words

\begin{tabular}{|c|c|c|c|}
\hline \multicolumn{2}{|c|}{ Noun phrases } & \multicolumn{2}{|c|}{ Compound nouns } \\
\hline Group A & Group B & Group A & Group B \\
\hline Ushi no takusii & Ushi no basu & Usagi takusii & Usagi basu \\
\hline Inu no takusii & Inu no basu & Iruka takusii & Iruka basu \\
\hline Ari no takusii & Ari no basu & Ahiru takusii & Ahiru basu \\
\hline Tora no takusii & Tora no basu & Tomato takusii & Tomato basu \\
\hline Kani no takusii & Kani no basu & Kaeru takusii & Kaeru basu \\
\hline Kuri no takusii & Kuri no basu & Kujira takusii & Kujira basu \\
\hline Same no takusii & Same no basu & Sakana takusii & Sakana basu \\
\hline
\end{tabular}


as representative in terms of the ranges of behaviors, were analyzed by a second trained judge. The rate of agreement in the identification of stuttering was 96.4\%. Differences between the two raters were resolved by the first author, listening repeatedly to the samples in question and making a final judgement, as in Natke, Grosser, Sandrieser, and Kalveram (2002).

\section{Results}

Figure 1 showed the frequency of stuttering in noun phrases and compound nouns. The frequency of stuttering was $14.8 \%(S D=20.7)$ with noun phrases and $19.9 \%$ ( $S D=23.6)$ with compound nouns. The frequency of stuttering was transformed to arcsine values before statistical comparisons were conducted. The frequency of stuttering in noun phrases was not significantly higher than in compound nouns $(t(13)=1.88, p>.05)$.

Each participant's frequency of stuttering in noun phrases and compound nouns was shown in Fig. 2. The participant's data are arranged in the order of the frequency of stuttering on the total number of stimuli. The only two children whose frequency of stuttering in noun phrases tended to be higher than in compound nouns were Child C and J. In contrast,

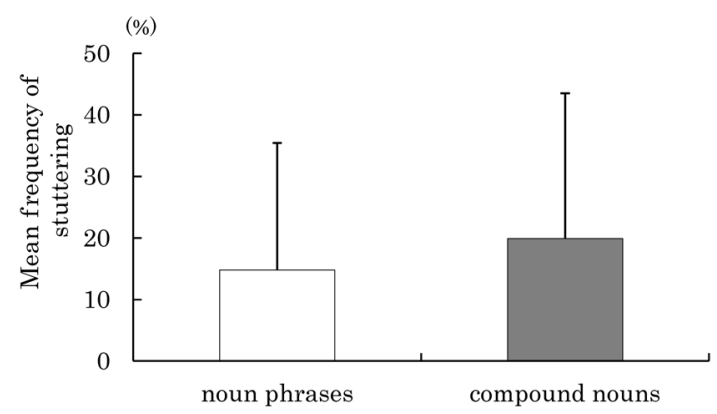

Fig. 1 Mean Frequency of Stuttering in Noun Phrases and Compound Nouns

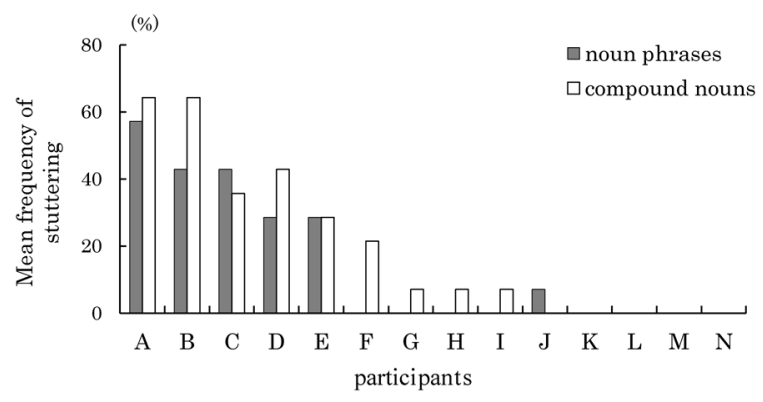

Fig. 2 Mean Frequency of Stuttering in Each Child there were seven children whose frequency of stuttering in compound nouns tended to be higher than in noun phrases (Child A, B, D, F, G, H, and I). The frequency of stuttering in noun phrases was equal to that in compound nouns in one child (Child E). Four children stuttered neither in noun phrases nor compound nouns (Child $\mathrm{K}, \mathrm{L}, \mathrm{M}$ and $\mathrm{N}$ ).

Figure 3 reveals the frequency of stuttering for the first and second elements of both noun phrases and compound nouns. The frequency of stuttering in the first element was $13.3(S D=18.8)$ with noun phrases, and $18.4 \%(S D=23.2)$ with compound nouns. In the second element, the frequency of stuttering was 1.5 $(S D=5.7)$ with noun phrases, and $1.5(S D=4.1)$ with compound nouns. A two-factor ANOVA was conducted. The frequency of stuttering was transformed to arcsine values before statistical comparisons were conducted. The frequency of stuttering in the first element was significantly higher than that in the second element $(F(1,13)=10.11, p<.01)$. The type of stimulus by the type of element interaction was not significant $(F(1,13)=2.84, p>.05)$.

\section{Discussion}

The purpose of the present study was to investigate whether the frequency of stuttering in noun phrases was significantly higher than in compound nouns for Japanese school-aged children who stutter. The results showed that the frequency of stuttering in noun phrases was not significantly higher than in compound nouns. Moreover, individual data also showed only two among fourteen children whose frequency of stuttering in noun phrases tended to be higher than that in compound nouns. Additionally, there were no significant differences in the loci of stuttering between noun phrases and compound nouns.

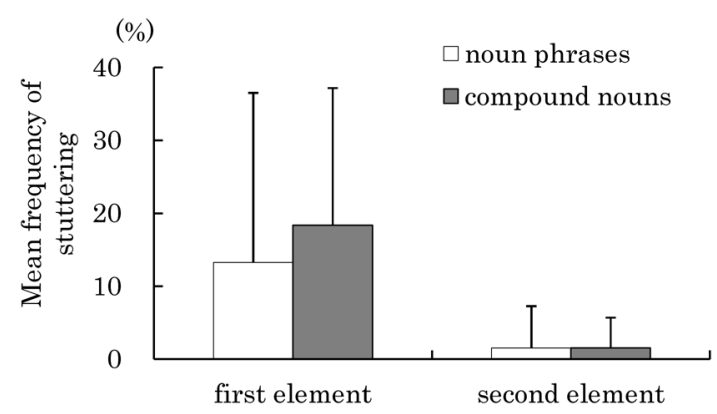

Fig. 3 Mean Frequency of Stuttering: Comparison Between the First and the Second Element 
These findings suggest that phrase-level processing is not more difficult than the word-level processing in school-aged children who stutter, and that schoolaged children who stutter do not experience difficulty with the processing of syntactic structures as we hypothesized.

It has been noted that the occurrence of stuttering in young children is closely related to the difficulty with processing of syntactic structure (Buhr \& Zebrowski, 2009; Gaines, Runyan, \& Meyers, 1991; Ratner \& Sih, 1987), whereas in adolescent or adults, it is not closely related to difficulty with processing of syntactic structures (Logan, 2001; Silverman \& Ratner, 1997). Based on these findings, the results of the present study suggest that the mechanism of linguistic processing in school-aged children who stutter resembles that in adolescents or adults rather than young children who stutter. Moreover, the present study also suggests that difficulty with processing of syntactic structures decreases before school-age. It is more likely that it is attributable to the development of linguistic processing in children who stutter as Bloodstein (2001) and Brocklehurst, Lickley, and Corley (2013) noted. In future investigations, it might be of interest to examine why the difficulty with syntactic processing decreases before school-age.

\section{References}

Bernstein, N. E. (1981) Are there constraints on childhood disfluency? Journal of Fluency Disorders, 6, 341-350.

Bloodstein, O. (2001) Incipient and developed stuttering as two distinct disorders resolving a dilemma. Journal of Fluency Disorders, 26, 67-73.

Bloodstein, O. \& Grossman, M. (1981) Early stutterings: Some aspects of their form and distribution. Journal of Speech, Language, and Hearing Research: JSLHR, 24, 298-302.

Brocklhurst, P. H., Lickley, R. J., \& Corley, M. C. (2013) Revisiting Bloodstein's anticipatory struggle hypothesis from a psycholinguistic perspective: A variable release threshold hypothesis of stuttering. Journal of Communication Disorders, 46, 217-237.

Buhr, A. \& Zebrowski, P. (2009) Sentence position and syntactic complexity of stuttering in early childhood: A longitudinal study. Journal of Fluency Disorders, 34, 155-172.

Gaines, N. D., Runyan, C. M., \& Meyers, S. C. (1991) A comparison of young stutterers' fluent versus stuttered utterances on measures of length and complexity. Journal of Speech and Hearing Research, 34, 37-42.

Logan, K. J. (2001) The effect of syntactic complexity upon the speech fluency of adolescents and adults who stutter. Journal of Fluency Disorders, 26, 85-106.

Natke, U., Grosser, J., Sandrieser, P., \& Kalveram, K. T. (2002) The duration component of the stress effect in stuttering. Journal of Fluency Disorders, 27, 305-318.

Ratner, N. B. \& Sih, C. S. (1987) Effects of gradual increases in sentence length and complexity on children's disfluency. The Journal of Speech and Hearing Disorders, 52, 278-287.

Sander, E. (1961) Reliability of the Iowa speech disfluency test. The Journal of Speech and Hearing Disorders, 7 (Suppl. 7), 2130.

Silverman, S. W. \& Ratner, N. B. (1997) Syntactic complexity, fluency, and accuracy of sentence imitation in adolescents. Journal of Speech, Language, and Hearing Research: JSLHR, 40, 95-106.

Takahashi, S. \& Ito, T. (2012) Effect of syntactic structure on the frequency of stuttering in Japanese children who stutter: Comparison between relative and coordinate clause. The Japan Journal of Logopedics and Phoniatrics, 53, 33-36. (in Japanese)

Wingate, M. E. (1964) A standard definition of stuttering. The Journal of Speech and Hearing Disorders, 29, 484-489. 